\title{
A Study of Digit Recognition Algorithm for Meter based on Rough Set and Neural Network
}

\author{
Xiaochen Zhang ${ }^{1}$, Yuanchang Zhong ${ }^{1,2^{*}}$, Jiajia Shen ${ }^{1}$, Kun Li and Congjun Feng ${ }^{1}$ \\ ${ }^{1}$ College of Communication Engineering, Chongqing University, \\ Chongqing, 400044, China \\ ${ }^{2}$ School of Automation, Chongqing University, Chongqing, 400044, China \\ \{20131202030,zyc, 20141202037,20141202025,20141213062\}@cqu.edu.cn
}

\begin{abstract}
Due to the low recognition accuracy, the remote meter reading technology based on camera direct reading has been developed slowly. Althoughthere is a variety of features data for recognizing digit in image using BP neural network, some of auta) cannot be used to recognize digit accurately. Moreover, the BP network has a slow rate of convergence, low accuracy and easily fall into local minimum. To solve the above questions, a new digit recognition algorithm of meter based on rough set and neyral network which are optimized by genetic algorithm is proposed. The improyed genetic rough set algorithm is used for reducing the data, and then the minimum featureattribute sets after reduction are input to genetic neural network for identifying digit. The experimental results show that the algorithm can effectively reduce the number of decision attributes and simplify the structure of the neural network with high 1 dentification accuracy and short training time, which improve the generdidation ability and robustness of the neural network.
\end{abstract}

Keywords: digit recognition; genette algorithm; rough set; neural network; attribute reduction

\section{Introduction}

With the development of smart home, intelligent meter reading system has been paid great attention. The Internet of things is prompting the coming of the era of "Internet of meters" which unites the household gas meter, water meter and electric meter. In the large-scale data statistics, the digit recognition of the meter is a very important part of the whole intelligent "nternet of meters", so it has become a hot research topic for many years $[1,2]$ Digit recognition generally uses the traditional methods of feature matching and feature discrimination, but the recognition rate of these methods is not high [35] With the rapid development of neural network technology, it itself has a high degree of parallelism, strong self-organizing ability and fault tolerance, and better noise interference suppression ability to open up a new route for rapid and accurate digit recognition $[6,7]$. However, neural network has a lot of local extreme points because of highly complex nonlinear structure and its training process is easy to fall into local extremum under large amounts of data. It not only seriously affects the convergence speed, but also may lead to training error converge to local optimal solution rather than global optimal solution, which will impact on the performance of the neural network seriously [8, 9]. Moreover, if the feature data redundancies are too many, it easily results in longer training time of BP neural network, overtraining and even completely unable to train.

Rough set theory can extract the characteristic more accurately as a powerful data analysis theory in the process of data preprocessing. In the process of data preprocessing, it can reduce the dimensions of the data set by using the reduction attribute of the rough set theory. In the rough set theory, the attribute reduction is mainly under the premise of keeping the classification ability of information system, deletes these unnecessary, 
unimportant or unrelated redundant attributes of the original decision system, so that achieves the purpose of simplifying the data $[10,11]$. Rough set theory can effectively remove large amounts of redundant information existing in the training samples, and extract important feature so as to reduce the vector dimensions of sample data and the training time of neural network [12].The existing reduction algorithm, mainly from the nucleus of rough set, uses heuristic search method to construct a reduction which contains the minimum condition attributes, but the scope of application of this algorithm is limited and complex [13].

On the basis of natural selection and genetic theory, genetic algorithm is an efficient global optimization search algorithm that combines the survival of the fittest in the process of biological evolution with random information exchange mechanism of group internal chromosomes. It can automatically acquire and accumulate knowledge about search space in the process of search, and adaptively control the search process in order to achieve the optimal solution $[14,15]$. Genetic algorithm combined with neurap network, also known as the genetic neural network. It not only can effectively paly the generalization and mapping ability of neural network but also overcome the shortcomings of slow convergence speed and easy to fallinto local minimum point of neural network [16]. Genetic reduction algorithm is an algorithm that genetic algorithm optimizes the attribute reduction of the rough set. It can efficiently reduce the computational complexity and can quickly converge to global optrmal solution.

For the digit recognition of no-pointer meter (take the gas meter for example), using reduction ability of rough set for data and BP neural network with good fault tolerance and adaptive ability in character recoghition, an algøithm based on rough set and neural network which are optimized by Genetic Algorithm(GA) is proposed in this paper. This algorithm can not only accurately) extract key characteristics of digit to reduce computational complexity, and shorten the structure of the neural network, but also can make the neural network eonverge to the global optimal solution quickly, overcome the defects of BP network effectively and improve the training speed and recognition precision.

\section{The Basic Concepts of Rough Set Theory}

Rough set theory is atheory about data analysis and inference, which is proposed by the academician Z.Pawlak of Poland in 1982. This theory does not need quantity description with certain characteristics or attributes given in advance and can effectively analyze and deal with imprecise, incomplete and inconsistent data. It also can find the hidden relationships between the data, discover the dependencies between objects and attributes, evaluate the importance of attributes for classification and extract useful data to simplify the information $[10,11]$. In order to construct the training sample and testing sample of classification algorithm based on genetic rough set for meter character recognition, we introduce the following definitions of the rough set.

Definition $1 \mathrm{~S}=(U, A, V, f)$ is called an information system, where:

(1) $U$ is universe, namely the non-empty finite set of objects;

(2)A is the non-empty finite set of attributes;

(3) $\mathrm{V}=U \mathrm{~V}_{\alpha}, \forall \alpha \in A$ is the range of information function;

(4) ${ }^{f}$ is the information function of information system;

If the attribute $\mathrm{A}$ can be divided into the condition attributes $\mathrm{C}$ and the decision attributes $\mathrm{D}$, that is $C \cup D=A, C \cap D=\varnothing$, then $\mathrm{S}$ is called the decision system or the decision table. 
Definition 2 Given an information system $\mathrm{S}=(U, A, V, f)$, for any one attribute subset $x_{i}, x_{j} \in U$, the indiscernibility relation $I N D(P)$ on the attribute subset $\mathrm{P}$ is defined as:

$\operatorname{IND}(P)=\left\{\left(x_{i}, x_{j}\right) \mid x_{i} \in U, x_{j} \in U, \forall \alpha \in P, f\left(x_{i}, \alpha\right)=f\left(x_{j}, \alpha\right)\right\}$

Obviously, $I N D(P)$ meets the reflexivity, symmetry and transitivity, is an equivalence relation on $\mathrm{U}$. We commonly use $\mathrm{U} / \mathrm{P}$ as the set constituted by all the equivalence classes produced by the indiscernibility relation $I N D(P)$. For $\forall x \in \mathrm{U}, \quad[x]_{I N D(P)}=[x]_{\mathrm{P}}$.

Definition 3 Given a decision system $\mathrm{S}=(U, C \cup \mathrm{D}, \mathrm{V}, f)$, an equivalence relation $\mathrm{R} \in I N D(K)$ on $\forall \mathrm{X} \subseteq \mathrm{U}$ and universe $\mathrm{U}$, the lower approximations of subset $\mathrm{X}$ with respect to $\mathrm{R}$ is defined as:

$\underline{\mathrm{R}}=\left\{x \mid(\forall x \in \mathrm{U}) \wedge\left([x]_{R} \subseteq \mathrm{X}\right)\right\}$

The lower approximations $\underline{R}$ is also called as R-positive region of $\mathrm{X}$ with respect to $\mathrm{R}$, which is written for $\operatorname{pos}_{R}(X)$ and $\operatorname{pos}(X)=\underline{R}(X)$

Definition 4 Given an information system $S=(U, A, V, \mathcal{Y}, P, Q \subseteq A, R \in P$, if $\operatorname{IND}(P)=I N D(P-\{\mathrm{R}\})$

we called $R$ is unnecessary in $P$, otherwise $R$ is necessary. If every $R \in P, R$ is necessary in $\mathrm{P}, \mathrm{P}$ is independent, otherwise $\mathrm{P}$ is dependent or not independent. Unnecessary attributes in the)information system are redundant and if removed from the system, it will not change the system classification ability.

Definition 5 Given an information system $\mathrm{S}=(U, A, V, f)$, a cluster of equivalence relations $\mathrm{P} \in \mathrm{A}$ on information system, for any $\mathrm{G} \subseteq \mathrm{P}$,if $\mathrm{G}$ is independent and $\mathrm{IND}(\mathrm{G})=\mathrm{IND}(P), \mathrm{G}$ is defined as a reduction of $\mathrm{P}$ and denoted as $\mathrm{G} \in \mathrm{RED}(\mathrm{P})$, where $\mathrm{RED}(\mathrm{P})$ is thecollection of all reduction components of $\mathrm{P}$.

For a cluster of equivalence relations $\mathrm{P}, \mathrm{Q} \subseteq \mathrm{A}, \in \mathrm{P}$, if $\operatorname{IND}(\mathrm{P})=\operatorname{IND}(P-\{\mathrm{R}\})$, a collection of all the necessary knowledge in $\mathrm{P}$ is called as core of $\mathrm{P}$ to describe it say CORE

\section{Rough Set Attribute Reduction Algorithm based on Genetic Algorithm Optimization}

Genetic reduction algorithm is an algorithm which is optimized for rough set attribute reduction algorithm by genetic algorithm for searching minimum relative reduction in the decision system. The minimum relative reduction is the attributes set containing the least number of conditional attributes in all the relative reduction of the attribute set. For a decision system, there's often more than one attribute reduction, the fewer number of condition attributes, the more simple of the decision system.

\subsection{The Selection of Fitness Function}

In the genetic algorithm, the fitness function is used to evaluate the quality of the individuals in the group. Individuals with high fitness can be more likely to be inherited to 
the next generation relative to individuals with low fitness. The form of the fitness function directly determines the evolution behavior of group. According to the actual requirements of this project, our improved fitness function is as follows:

$$
F(x)=(1-\alpha) f(x)+\alpha h(x)=(1-\alpha)\left(1-\frac{\operatorname{card}(x)}{\operatorname{card}(C)}\right)+\alpha \frac{\operatorname{card}\left(\operatorname{pos}_{x}(D)\right)}{\operatorname{card}\left(\operatorname{pos}_{c}(D)\right)}
$$

This function consists of two parts, the first part $f(x)$ is the objective function, where card (x) is numbers of 1 in individual $x$. card $(C)$ is numbers of the condition attribute $\mathrm{C}$. The meaning of $f(x)$ is the proportion of attributes that not include individual $x$. The second part $h(x)$ is penalty function, which is the proportion of condition attribute $\mathrm{C}$ to the decision attribute $\mathrm{D}$ in an individuat. proportional coefficient. The main function of $f(x)$ is mâke the condition numbers in $x$ as little as possible, the main function of $h(x)$ is to thake the decision attribute support for condition in $x$ as large as possible. Therefore, this fitness function satisfies the condition for obtaining minimum relative reduction.

\subsection{The Implementation Process of the Genetic Reduction Algorithm}

The process of genetic reduction algørithm based attribute dependency:

Input information: the decis $\mathrm{n}$ ) system $\mathrm{S}=(U, \mathrm{C} \cup D, V, f)$ after continuous attribute discretization.

Output information: the decision system of attribute reduction.

(1) Calculate the dependency of decision attributes D relative to condition attributes C ;

(2) Calculate the attribute core $\operatorname{CORE}(C)$, let $\operatorname{CORE}(C)=\varnothing$, for $\alpha \in C$, if $r(C-\{\alpha\}, D)=r(C, D), C O R E(C)$ is the minimum relative reduction.

(3) Generating-initial population, randomly generating $t$ initial population that be composed of individuals represented by the binary string whose length is $m$ (the number of conditional attributes). For the attributes in the core, the corresponding bit is 1 , and the other bits are randomly selected from 0 or 1 .

(4) Calculate the fitness value. For each chromosome in population, calculate the dependence of the decision attribute for the condition attribute for each individual. Calculate the fitness of each individual according to the fitness function $F(x)$.

(5) Selection operation. Calculate the probability of each individual that is selected by way of roulette wheel, then construct a new population.

(6) Crossover operation, according to the crossover probability ${ }^{P_{c}}$, makes crossover operation for population by way of single point crossover, then obtain a new population made of new chromosomes .

(7) Mutation operation, according to the mutation probability ${ }^{P_{\mathrm{m}}}$, make mutation operation for population by the way of basic bit mutation, then obtain a new population. 
(8) Modification and check, according to the optimal preservation strategy, make the best individual copy to the next generation, and save the best individual.

(9) Termination conditions, judge whether satisfy the end condition, if the algorithm is not satisfied, then return (4) and continue to iteration.

\section{BP Neural Network based on Genetic Algorithm Optimization}

\subsection{The Basic Principles of Genetic Neural Network}

In the combination of genetic algorithm and BP neural network, there are two main forms: one is under the condition of the fixed network topology, using genetic algorithm to optimize the weights of neural network; Another is using genetic algorittim to optimize the network topology, and then to optimize the network parameters. For digit ecognition system of this paper, we can determine the BP neural network topology/through repeated network test, so this paper optimizes the initial weights and thresholds of the neural network by genetic algorithm.

\subsection{The Learning Process based on Genetic Algorithm to Optimize the Weights and} Thresholds of Neural Network

In this paper, we use three layers BPnetwork. $I_{i}$ is the output of the ${ }^{i}$ th node in the input layer. ${ }^{H_{i}}$ is the output of the ${ }^{i}$ th node in the hidden layer. ${ }^{O}{ }_{i}$ is the output of the ${ }^{i}$ th node in the output layer. $W I H_{i j}$ is the connection weight between the $i$ th node in the input layer and the th node in the hidden layer. ${ }^{W H} O_{j t}$ is the weight between the ${ }^{j}$ th node in the hidden layer and the ${ }^{t}$ th node in the output layer. GA-BP algorithm flow chart is shown in Figure 10

According to the process of GA-BP algorithm, combined with the optimization of the initial parameters of BP network, the following specific steps can be obtained:

(1) Initialization population $P$. Initialize crossover scale, crossover probability ${ }^{P_{c}}$, mutation propability $P_{\mathrm{m}}$ and any $W I H_{i j}$ and $W H O_{j t}$. In the encoding, using binary encoding, the mitial population is 40 in this paper.

(2) Carculate the fitness function of each individual, choose network individual according to the following probabilities:

$$
P_{s}=\frac{f_{i}}{\sum_{i=1}^{N} f_{i}}
$$

where $f_{i}$ is the fitness value of the individual $i$, which is measured by error square sum $E$, namely

$$
f(i)=\frac{1}{E(i)}
$$




$$
E(i)=\sum_{p=1}^{m} \sum_{k=1}^{q}\left(V_{k}-T_{k}\right)^{2}
$$

where ${ }^{i=1, \cdots, \mathrm{N}}$ is the chromosome number. $k=1, \cdots, q$ is the node number in the output layer. $p=1, \cdots, m$ is the learning sample number. $V_{k}$ is the desired output of the network. $T_{k}$ is the actual output of the network.

(3) Crossover operation for individuals $G_{i}$ and $G_{i+1}$ by using the cross probability $P_{c}$ to generate new individuals $G_{i}^{\prime}$ and $G_{i+1}$. Those without the crossover operation are directly copied.

(4) Mutation operation for individual ${ }^{G_{j}}$ by using the mutation probability $P_{\mathrm{m}}$ to generate a new individual $G_{j}{ }_{j}$.

(5) Insert the new individual into the population $\mathrm{P}$, and calculate the fitness function of the new individual.

(6) To determine whether the algorithm (is over, if find a satisfactory individual, then the end, otherwise go to step (3).

Algorithm is over, if reaching preset performance index, the optimized network connection weight can be obtained after decoding from the best individual in the final group.

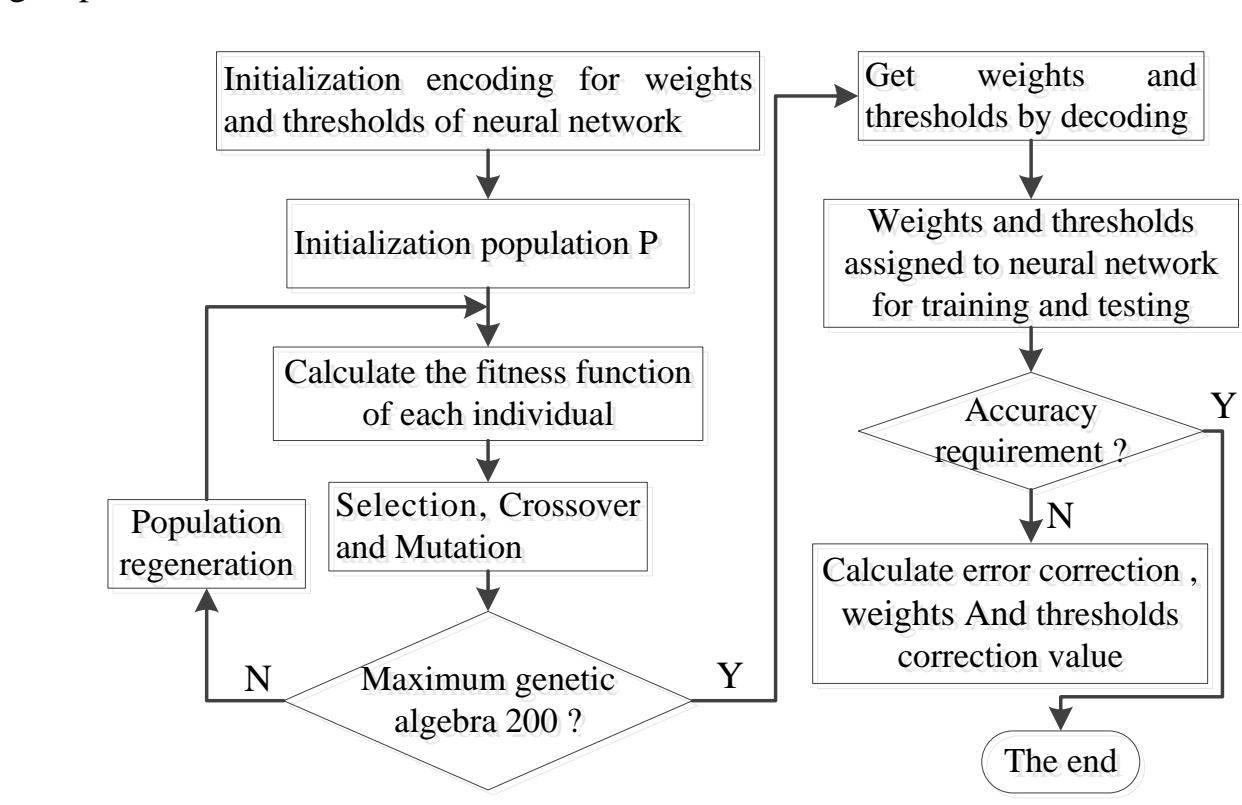

Figure 1. GA-BP Algorithm Flow Diagram

\section{The Simulation Experiment and Result Analysis of Digit Recognition for Meter Image}

To verify the validity of our improved algorithm in this paper, we firstly preprocess the collected gas meter image and extract the feature vectors of digit characters. However, if 
directly put these feature vectors as the input of the neural network, it will undoubtedly become sharp complex and large of the network, and prolong the training time. After extracting features, in order to enhance the recognition efficiency and accuracy of the network, we remove redundant characteristics to input those key information which best reflect the digit characteristics into the neural network. Therefore, we begin to construct the decision system of rough set after extracting feature, and use genetic rough set for attribute reduction of decision system, then divide minimum feature attribute set after reduction into training samples and testing samples, and make those sample data input genetic neural network which is used for recognizing the digit and then output the result. The system model of meter image recognition based on rough set and neural network optimized by genetic algorithm is shown in Figure 2.

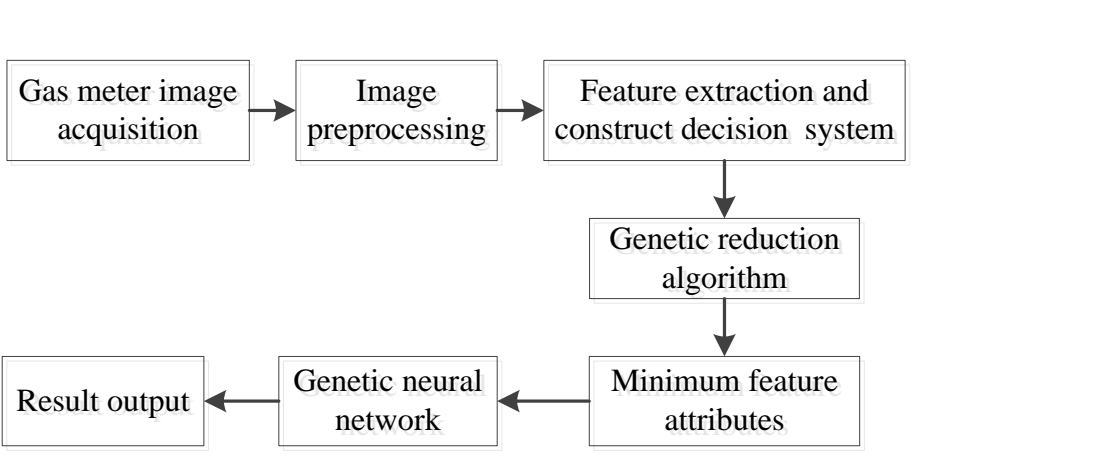

Figure 2. System Model of Meterlmage Recognition based on Rough Set and Neural Network Optimized by Genetic Algorithm

\subsection{Image preprocessing}

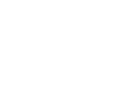

Collect gas meter dial hages in the actual environment. However, for some reasons, the collected images are conducive to identify digit accurately, such as camera installation tilt or the non-uniform and fuzzy brightness of the images. Image preprocessing is used for enhancing images and eliminating the noise of images and binarization processing, using the filter technique to eliminate the noise which is inevitably introduced during the image acquisition process. Extracting information by binarization, the target and background will be separated to improve image quality for the subsequent recognition $[17,18]$.

These 60 gas meter dial images are chose in different light, different angle and different definition, and then respectively adding $0.1,0.2,0.3$ noise to produce a total of 240 initial samples. The target images are segmented by the tilt correction and the region location. Through binarization processing and filtering, and then the combination of vertical and horizontal projection and normalization method to segment to produce each size of $44 \times 28$ character image. Character images are thinned by the Roberts edge detection operator for reducing the number of pixels. Each gas meter dial image extract 7 digit characters and 1600 are randomly selected from all digit characters, one of 1200 as the training sample, 400 as testing samples. Part of the process of image preprocessing is shown in Figure 3. The image preprocessing is not the key that we study in this paper, so we don't do detailed descriptions. 


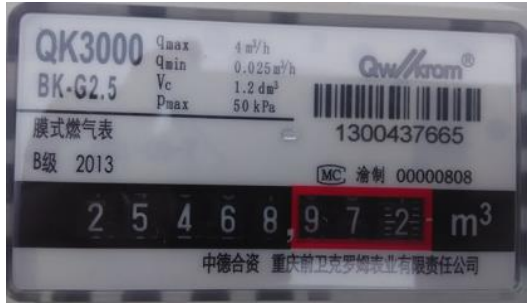

(a) Initial gas meter image

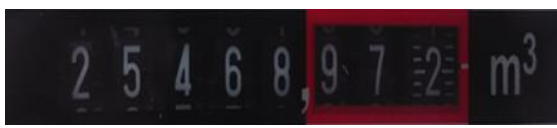

(b) Target image after locating and segmenting

\section{$25468,9 \cdot 7 \Rightarrow \mathrm{m}^{3}$}

(c) Image after binaryzation and tiltering

\section{7}

(d) $44 \times 28$ images after segmentation and normalization (e) $44 \times 28$ images after thinning

Figure 3. Part of the Process of Image Preprodessing

\subsection{Character Feature Extraction}

Feature extraction is the first step on the patten recognition and its goal is to get a vector $\left\{\mathrm{t}_{1}, \mathrm{t}_{2}, \cdots, \mathrm{t}_{n}\right\}$ which is used to represent the original digital characters, so the selection of feature extraction method is animportant factor that affects the recognition rate.

Considering the contact of recoghition accuracy and eigenvector dimension and the need of experiments this paper adopts many feature extraction methods to extract the feature vector as training and testing samples of the neural network. The coarse grid feature extraction is used to segment $44 \times 28$ digit images into $2 \times 7$ small area which number is 88 , and then $c$ a eulate the percentage of image pixels in every area. The pixel ratio feature extraction is used to calculate digit image pixels as a percentage of all pixels of images. The structure feature extraction is to extract the number of intersections in the vertical midline, Nertical $1 / 4$, vertical $3 / 4$, horizontal midline, horizontal $1 / 3$, horizontal $2 / 3$, leading diagonal and minor diagonal, obtaining a total of 8 structure features. The stroke density features is to scan digit in different directions and every 4 rows of longitudinal and transverse is scanned respectively, obtaining a total of 18 feature values. Consequently, a total of 115 dimensional feature vectors represent every one digital image.

\subsection{Attribute Reduction of the Feature Vector based on Genetic Rough Set}

The universe $U$ includes the total number of 1600 characters. The condition attribute $\mathrm{C}=\left\{\mathrm{c}_{1}, \mathrm{c}_{2}, \cdots, \mathrm{c}_{115}\right\}$ includes 88 dimensional coarse grid, 1 dimensional pixel ratio, 8 dimensional structural features and 18 dimensional density features. The decision attribute $D=\{0,1, \cdots, 9\} \quad$ is corresponding to the 10 numbers. Relative reductions $\left\{\mathrm{c}_{46}, \mathrm{c}_{79}, \mathrm{c}_{82}, \mathrm{c}_{89}, \mathrm{c}_{91}, \mathrm{c}_{94}, \mathrm{c}_{97}, \mathrm{c}_{106}, \mathrm{c}_{112}\right\}$ which are the minimum condition attribute relative to decision attribute $D$ are obtained by genetic reduction algorithm, where include 3 dimensional coarse grid, 1 dimensional pixel ratio, 3 dimensional structural 
features and 2 dimensional density features. Part of the reduction results are shown in table 1 .

Table 1. Part of the Reduction Results of Meter Digital Feature Vector

\begin{tabular}{|c|c|c|c|c|c|c|c|c|c|c|}
\hline sample & $c_{46}$ & $c_{79}$ & $c_{82}$ & $c_{89}$ & $c_{91}$ & $c_{94}$ & $c_{97}$ & $c_{106}$ & $c_{112}$ & digit \\
\hline 1 & 0.183 & 4 & 4 & 5 & 0.286 & 0.357 & 0.643 & 0.170 & 0.091 & 0 \\
\hline 2 & 0.093 & 6 & 3 & 3 & 0.214 & 0.286 & 0.357 & 0.071 & 0.017 & 1 \\
\hline 3 & 0.171 & 7 & 3 & 3 & 0.357 & 0.571 & 0.214 & 0.125 & 0.210 & 2 \\
\hline 4 & 0.182 & 5 & 2 & 6 & 0.286 & 0.429 & 0.071 & 0.161 & 0.120 & \multirow{3}{*}{3} \\
\hline 5 & 0.134 & 4 & 3 & 4 & 0.571 & 0.357 & 0.714 & 0.089 & 01182 & 4 \\
\hline 6 & 0.179 & 5 & 8 & 5 & 0.500 & 0.286 & 0.571 & 0.107 & 0.131 & 5 \\
\hline 7 & 0.188 & 3 & 2 & 6 & 0.357 & 0 & 0.214 & 0.22 & 0.108 & 6 \\
\hline 8 & 0.122 & 1 & 2 & 4 & 0 & 0.286 & 0.286 & 0.080 & 0.222 & 7 \\
\hline 9 & 0.190 & 4 & 5 & 6 & 0 & 0.643 & 0.429 & 0.179 & 0.125 & 8 \\
\hline 10 & 0.184 & 8 & 4 & 5 & 0.357 & 0.429 & 0.214 & 0.205 & 0.125 & 9 \\
\hline
\end{tabular}

\subsection{Digit Recognition based on the Genetic Neural Network}

The three layer BP neural networl is chosen which includes the input layer, the hidden layer and the output layer. To verify the superiority of our algorithm, the number of neurons in the input layer chooses 115 and 9 respectively, corresponding to the feature vectors of digits. The number of neurons in the output layer selects 10, adopting the position order of " 1 ". respectively, that is the encoding way of 0000000001,1000000000 , $\ldots, 0000000010$ on behalf of ten digits which is $0,1, \ldots, 9$. Too many neurons in the hidden layer can equse learning time is too long and too little may lead to poor fault tolerance and tow recognition ability for without the training samples. Taking all factors as a whole and the need of our algorithm, we find that recognition rate is the highest when the number of neurons in the hidden layer is 10 after repeated training testing and error comparison. Therefore, the number of neurons in the hidden layer takes 10.

To verify reoognition accuracy and training speed of our improved algorithm, the maximum training time of the neural network takes 5000, the performance index of training error is set to 0.001 and maximum hereditary algebra is 200 . The error of the output is determined by the following formula:

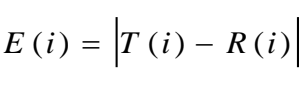

where $i=1,2, \cdots, 400$ is test sample serial number of the neural network; ${ }^{T(i)}$ is the actual output; $R(i)$ is the desired output.

We input the training samples into BP neural network, the network made up of rough sets and BP network, the network made up of rough sets and genetic neural network, and the network made up of genetic rough set and genetic neural network, respectively. Network is trained until the output error of network meets the convergence criteria or meets preset conditions, and the training error curve diagrams of four kinds of neural networks are shown in Figure 4. 


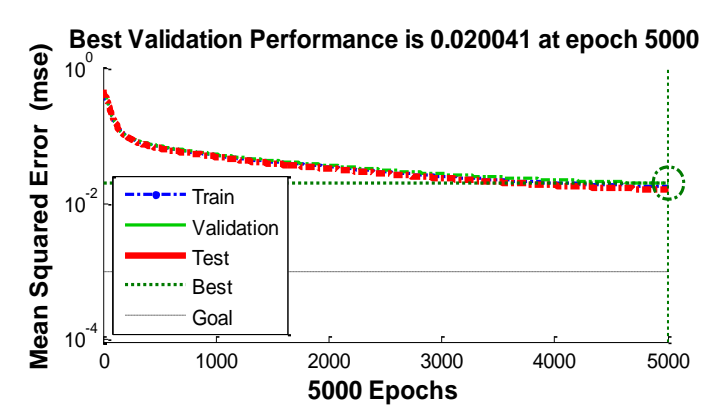

(a) BP neural network

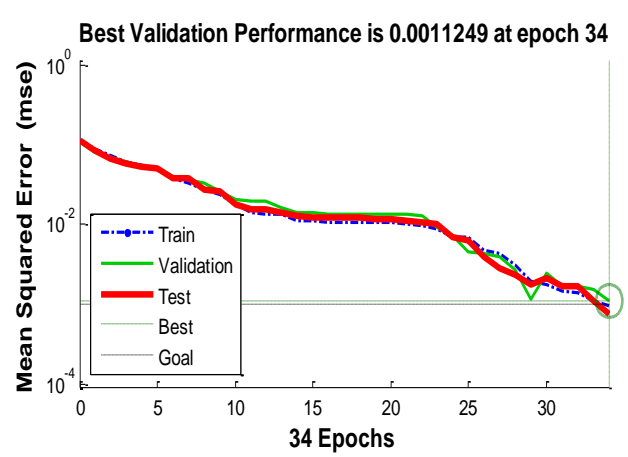

(c) rough sets and genetic neural network

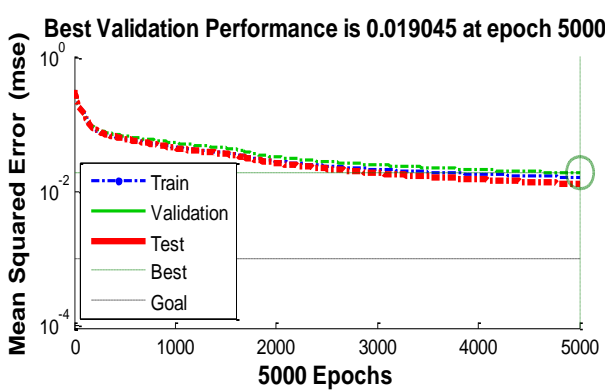

(b) rough sets and BP network

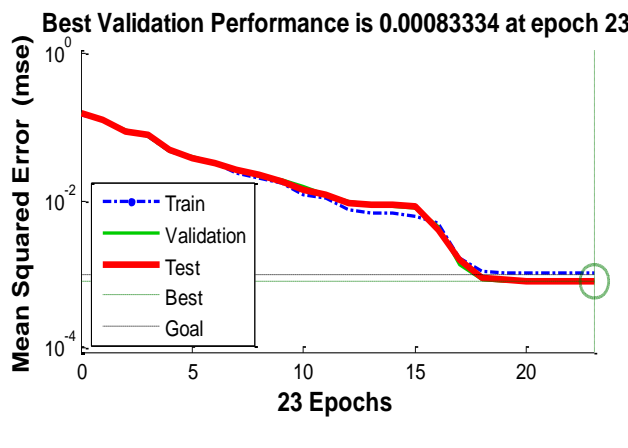

(d) rough set and neural network optimized by genetic algorithm

\section{Figure 4. Training Error Curve Diagram of Kour Kinds of Neural Networks}

The error curves of output or each neurat network are obtained by formula (8) which are shown in Figure 5 and output the recognition rate. Structure parameters, iterative times until achieve the goal by training, training time and recognition accuracy of each neural network areshownin table 2.

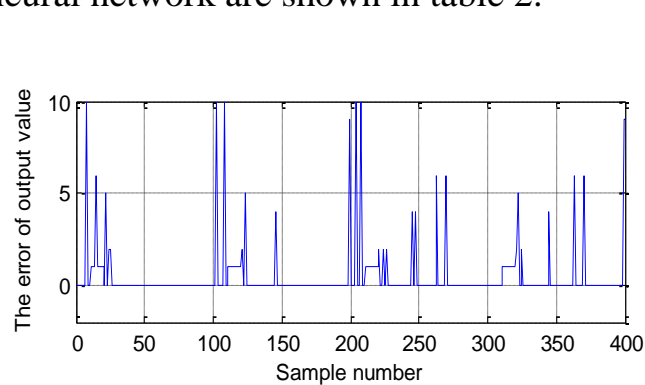

(a) BP neural network

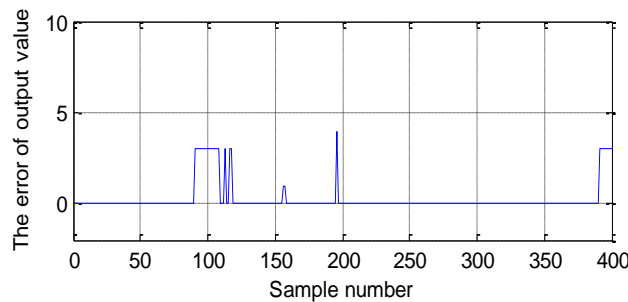

(c) rough sets and genetic neural network

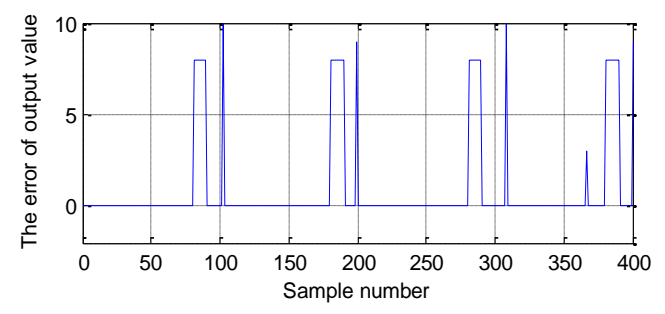

(b) rough sets and BP network

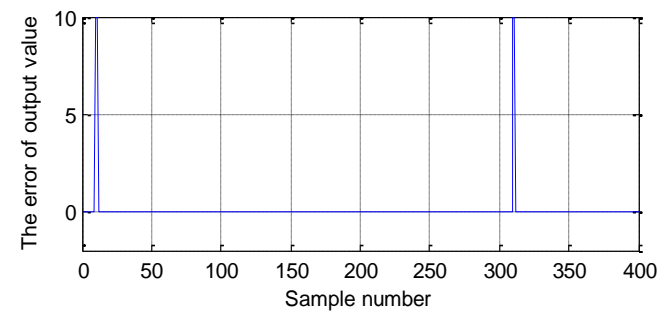

(d) rough set and neural network optimized

by genetic algorithm

Figure 5. Error Curve of the Output of Four Kinds of Neural Networks

Table 2. Comparisons of Four Kinds of Neural Network Parameters and Recognition Results 


\begin{tabular}{|c|c|c|c|c|}
\hline Type & $\begin{array}{c}\text { BP } \\
\text { network }\end{array}$ & $\begin{array}{c}\text { rough sets } \\
\text { and BP } \\
\text { network }\end{array}$ & $\begin{array}{c}\text { rough sets and } \\
\text { genetic neural } \\
\text { network }\end{array}$ & $\begin{array}{c}\text { rough set and neural } \\
\text { network optimized by } \\
\text { genetic algorithm }\end{array}$ \\
\hline input layer node & 115 & 9 & 9 & $\mathbf{9}$ \\
\hline Hidden layer node & 10 & 10 & 10 & $\mathbf{1 0}$ \\
\hline Output layer node & 10 & 10 & 10 & $\mathbf{1 0}$ \\
\hline $\begin{array}{c}\text { ultimate iterative } \\
\text { times }\end{array}$ & 5000 & 5000 & 34 & $\mathbf{2 3}$ \\
\hline $\begin{array}{c}\text { training time (s) } \\
\text { recognition } \\
\text { accuracy }\end{array}$ & 174.5879 & 129.6046 & 47.9241 & $\mathbf{2 3 . 8 7 0 9}$ \\
\hline
\end{tabular}

As can be seen from the above results, network training time based on roygh set and neural network which are optimized by genetic algorithm is the fastest and can quickly converge to the target value, and the network structure is simple and recognition accuracy is the highest. Even when repeated testing and training and performance index of training error continues to decrease, the recognition accuracy can reach $100 \%$.

\section{Conclusion}

In order to solve the camera direet reading forhousehold gas meter, a character recognition algorithm based on rough sets andeneural networks which are optimized by genetic algorithm is proposed in this paper. By means of attribute reduction of rough set simplifies feature vector of charadter and obtains the minimum feature attribute sets which input into the neural network to identify the digits. The whole process is optimized by genetic algorithm to effectively reduce the number of decision attribute, simplify the structure of the neurai network and avoid falling into a local minimum. Theoretical analysis and simulation results show that the algorithm can improve recognition performance with higher robustness, boost the anti-jamming capability and shorten the training time for the neural-network. It can be widely used for digit recognition of nonpointer instrument dial and can construct a universal character recognizer for household instrument, which opens up a new way for realizing smart home and Internet of meter.

\section{Acknowledgements}

This work is supported in part by the Major State Basic Research Development Program 973 (No. 2012CB215202).

\section{References}

[1] J. Gao and H. Ye, "An effective two-times recognition algorithm of meter digital image", Journal of Southeast University: Natural Science Edition, vol.43, (2013), pp. 153-157.

[2] K. D. T. M. Milanez and M. J. C. Pontes, "Classification of edible vegetable oil using digital image and pattern recognition techniques", Microchemical Journal, vol.113, (2014), pp. 10-16.

[3] W. N. Lu, C. R. Liu and Y.C. Zheng, et.al, "A Method for Digital Instrument Character Recognition Based on Template Matching", Modern Computer, vol.3, (2008), pp. 70-72.

[4] X. H. Xing and G. H. Gu, "Method of Quickly Recognizing Vehicle Plate Based on Pattern Matching and Characteristic Dot Matching", Optoelectronic Technology, vol.23, no.4, (2003), pp. 268-270.

[5] X. Chen, X. Liu and Y. Jia, "Discriminative Structure Selection Method Of Gaussian Mixture Models With Its Application To Handwritten Digit Recognition", Neurocomputing, vol.74, no.6, (2011), pp. 954-961.

[6] X. W. Li, S. J. Cho and S. T Kim, "Combined use of bp neural network and computational integral imaging reconstruction for optical multiple-image security", Optics Communications, vol.315, no.6, (2014). pp. 147-158. 
[7] J. Kasperkiewicz, J. Racz and A. Dubrawski, "HPC Strength Prediction Using Artificial Neural Network", Journal of Computing in Civil Engineering, vol.9, no.4,(2014), pp. 279-284.

[8] J. Zhou, W. Ma and J. Miao, "Fire Risk Assessment of Transmission Line Based on BP Neural Network", International Journal of Smart Home,vol.8, no.3, (2014), pp.119-126.

[9] G. Chen, K. Fu, Z. W. Liang, T. Sema, C. Li, P. Tontiwachwuthikul and R. Ldem, "The genetic algorithm based back propagation neural network for MMP prediction in CO 2 -EOR process", Fuel, vol.126, no.9, (2014), pp. 202-212.

[10] Z. Zhang and X. Yang, "Tolerance-based multigranulation rough sets in incomplete systems", Frontiers of Computer Science, vol.8, no.5, (2014), pp. 753-762.

[11] W. X. Zhang and W. Z. Wu, "An Introduction and a Survey for the Studies of Rough set Theory", Fuzzy Fystems and Mathematics, vol.14, no.4, (2000), pp. 1-12.

[12] H. Yu, Z. Liu and G. Wang, "An automatic method to determine the number of clusters using decisiontheoretic rough set", International Journal of Approximate Reasoning, vol.55, no.1, (2014), pp. 101-115.

[13] S. Y. Jing, "A hybrid genetic algorithm for feature subset selection in rough set theory", Soft Computing, vol.18, no.7, (2014), pp.1373-1382.

[14] S. D. Rajan, "Sizing, Shape, and Topology Design Optimization of Trusses Using Genetic Algonthm", Journal of Structural Engineering, vol.121, no.10, (2014), pp.1480-1487.

[15] M, Betti, L. Facchini L and P. Biagin, "Damage detection on a three-storey steel frame using artificial neural networks and genetic algorithms" Meccanica, vol.50, no.3, (2015) pp. 875,886

[16] L. Tian, Y. Luo and Y. Wang, "Prediction Model of TIG Welding Seam Size Based on BP Neural Network Optimized by Genetic Algorithm", Journal of Shanghay Jiaotong University, vol.47, no.11, (2013), pp.1690-1701.

[17] H. P. Corporation, "Efficient active contour and k-means algorithms in image segmentation", Scientific Programming, vol.12, no.2, (2014), pp.101-120.

[18] Q. Ga, J. Liu and H. Zhang, "Enhanced fisher discriminant criterion for image recognition", Pattern Recognition, vol.45, no.10, (2012), pp.3717-3724.

\section{Authors}

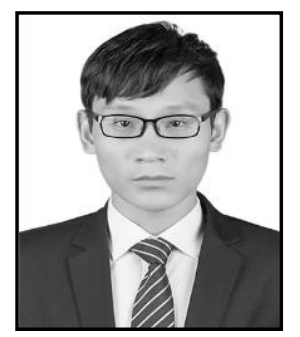

Xiaochen Zhang, he received the B.E degree in electronic information science and technology from Fuyang Teachers College in 2012 He is currently pursuing the M.S. degree in the College of Communication Engineering of Chongqing University, Chongqing, China. His $<$ research interests include pattern recognition and automatic Control.
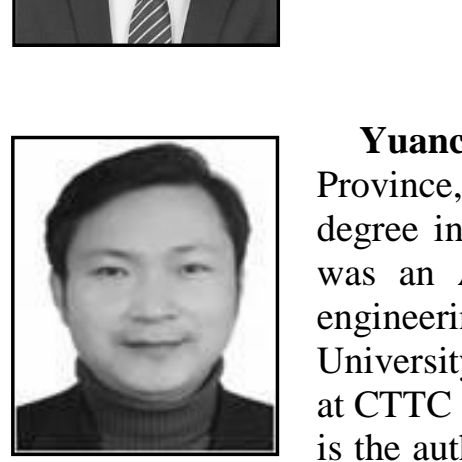

(2)

Yuanchang Zhong, he was born in Nanchong City, Sichuan Province, in 1965. He received M.S. degrees in 2004 and the Ph.D. degree in 2011 from Chongqing University.From 2003 to 2011, he was an Assistant Professor with the College of Communication engineering, since 2012, he has been a Professor at Chongqing University, where he leads the Networks and Mobile Systems group at CTTC (communication and tracking telemetering \& command). He is the author of two books, more than 100 articles, and more than 10 inventions. His research is in the area of communication and tracking telemetering \& command; Circuits and systems; wireless sensor networks. In addition to many widely cited papers, several systems developed as part of his research are available in the public domain. Some are in production or commercial use. 


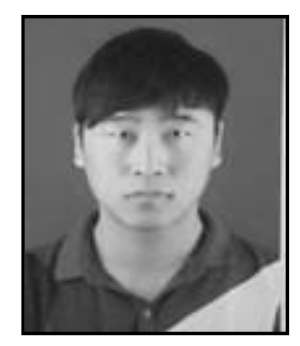

Jiajia Shen was born in Anhui Province, China, in 1992. He is currently pursuing the M.S. degree in the College of Communication Engineering of Chongqing University, Chongqing, China. His research interests include wireless monitoring and large data storage.

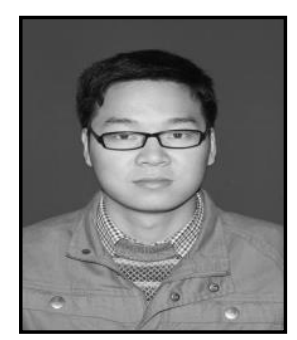

Kun Li was born in HeNan Province, China, in 1990. He is currently pursuing the M.S. degree in the College of Communication Engineering of Chongqing University, Chongqing, China. His research interests include pattern recognition and wireless monitoring.

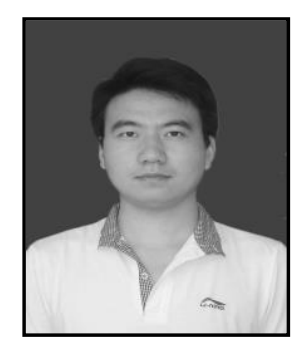

Congjun Feng was born in Henan Province, Chlina, in 1990. He is currently pursuing the M.S. degree in the College of Communication Engineering of Chongqing University, Chongqing, China. His research interests include internet of thitngs and pattern recognition. 
International Journal of Smart Home

Vol. 10, No. 8 (2016)

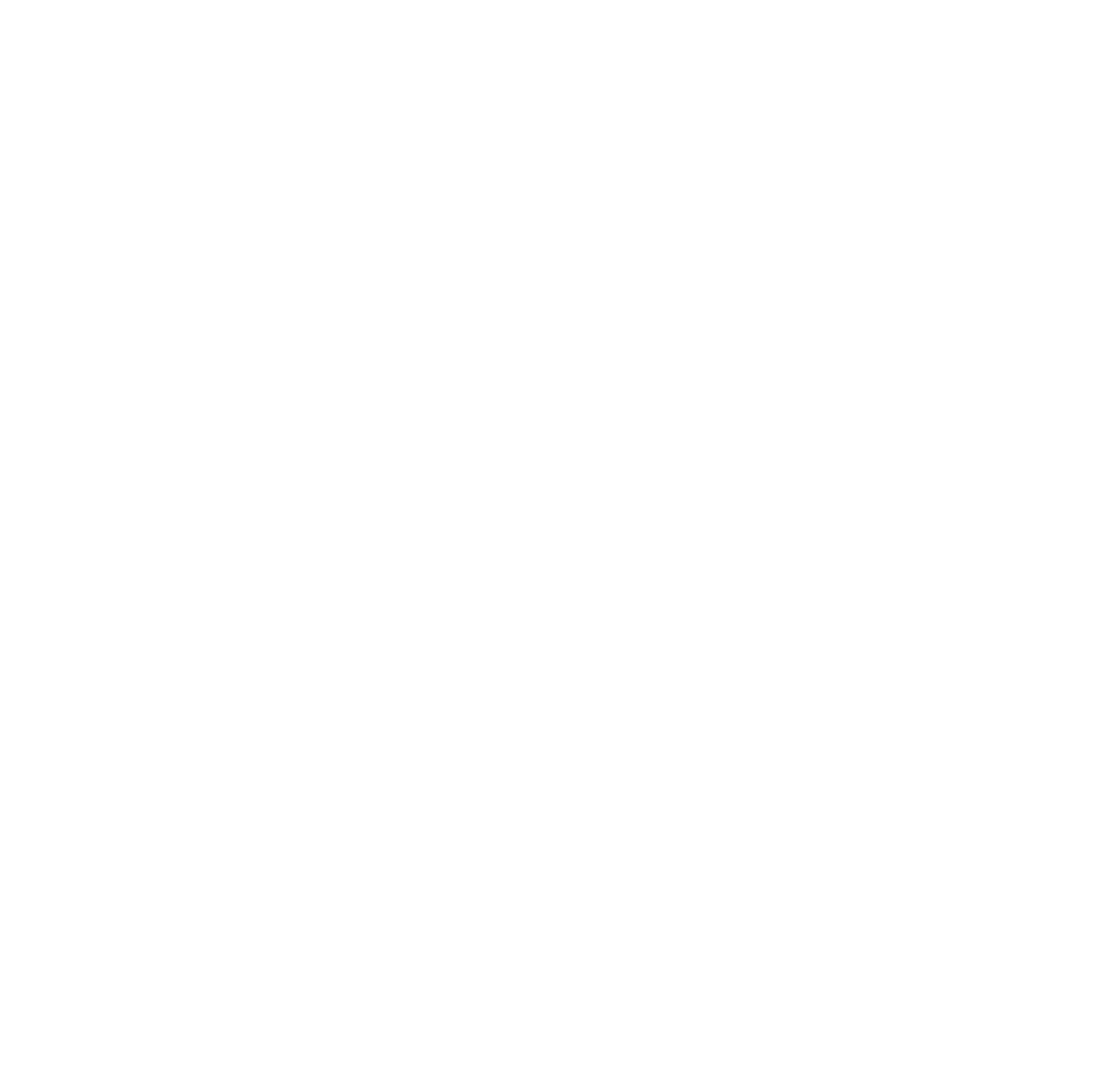

